\title{
A Look Back at Some Early Results in Membrane Computing *
}

\author{
Oscar H. Ibarra \\ Department of Computer Science \\ University of California \\ Santa Barbara, CA 93106, USA \\ Email: ibarra@cs.ucsb.edu
}

There have been tremendous research activities in the area of membrane computing initiated by Gheorghe Păun in a seminal paper [1] ten years ago (see also [2]). Membrane computing identifies an unconventional computing model, namely a $\mathrm{P}$ system, from natural phenomena of cell evolutions and chemical reactions. Due to the built-in nature of maximal parallelism inherent in the model, $\mathrm{P}$ systems have a great potential for implementing massively concurrent systems in an efficient way that would allow us to solve currently intractable problems in much the same way as the promise of quantum and DNA computing, once future bio-technology (or silicon-technology) gives way to a practical bio-realization (or chip-realization).

A $\mathrm{P}$ system is a computing model, which abstracts from the way the living cells process chemical compounds in their compartmental structure. The regions defined by a membrane structure contain objects that evolve according to specified rules. The objects can be described by symbols or by strings of symbols, and multisets of these objects are placed in the regions of the membrane structure. The membranes themselves are organized as a Venn diagram or a tree structure where one membrane may contain other membranes. By using the rules in a nondeterministic, maximally parallel manner, transitions between the system configurations can be obtained. A sequence of transitions shows how the system is evolving. Various ways of controlling the transfer of objects from a region to another and applying the rules, as well as possibilities to dissolve, divide or create membranes have been studied. $\mathrm{P}$ systems were introduced with the goal to abstract a new computing model from the structure and the functioning of the living cell (as a branch of the general effort of Natural Computing - to explore new models, ideas, paradigms from the way nature computes). Membrane computing has been very successful: many models have been introduced, most of them Turing complete and/or able to solve computationally intractable problems (NP-complete, PSPACE-complete) in a feasible time, by trading space for time; development of software and simulations; proposals for various potential applications. See the $\mathrm{P}$ system website

\footnotetext{
^ This research was supported in part by NSF Grant CCF-0524136.
} 
at http://ppage.psystems.eu/ for a large collection of papers in the area, and in particular the monograph [3].

On this tenth anniversary of the Workshop on Membrane Computing, it seems appropriate and fitting to look back at some early basic contributions in the area. In this talk, we will give a brief summary of results (mostly by the author and his collaborators: Zhe Dang, Andrei Păun, Gheorghe Păun, Hsu-Chun Yen, Sara Woodworth), some of which answered fundamental open questions in the field. These concern complexity issues such as universality versus non-universality, determinism versus nondeterminism, various notions of parallelism, membrane and alphabet-size hierarchies, and characterizations of some classes of $\mathrm{P}$ systems. We will also discuss some related open problems.

\section{References}

1. Gh. Păun. Computing with membranes. Turku University Computer Science Research Report No. 208, 1998.

2. Gh. Păun. Computing with membranes. Journal of Computer and System Sciences, 61(1):108-143, 2000.

3. Gh. Păun. Membrane Computing: An Introduction. Springer-Verlag, 2002. 